\title{
Valve exploiting the principle of a side channel turbine
}

\author{
Pavel Jandourek ${ }^{1, a}$, Vladimír Habán ${ }^{1}$, František Pochylý ${ }^{1}$ and Miloslav Fic ${ }^{1}$ \\ ${ }^{1}$ Brno University of Technology, Faculty of Mechanical Engineering, Energy Institute, Victor Kaplan Department of Fluid Engineering, \\ Technicka 2896/2, 61669 Brno, Czech Republic
}

\begin{abstract}
The article deals with a side channel turbine, which can be used as a suitable substitute for a pressure reducing valve. Reducing valves are a source of hydraulic losses. The aim is to replace them by a side channel turbine. With that in mind, hydraulic losses can be replaced by a production of electrical energy at comparable characteristics of the valve and the turbine. The basis for the design is the loss characteristics of the valve. Thereby creating a kind of turbine valve with speed-controlled flow in dependence of runner revolution.
\end{abstract}

\section{Introduction}

In many technical solutions, it is necessary to decrease the pressure of liquids. Pressure reducing valves are normally used for this pressure reduction. However, it utilizes a considerable part of the pressure energy that could transform into e.g. electric energy. Part of the pressure energy which is not used, could be transformed e.g. into electric energy. The pumping technique uses side channel pumps for pumping small quantities of liquids to large transport heights. These pumps are characterized by low-specific speed $\mathrm{n}_{\mathrm{s}}=4 \div 40 \mathrm{~min}^{-1}$ and after a small reconstruction they can be used in a turbine mode. It is possible to replace pressure reducing valves with side channel turbines. Thereby creating a new kind of turbine valve with speed-controlled flow dependent on the revolution of the runner.

At the pumped-storage power plant the water for cooling is taken directly from the penstock, see (Fig. 1.). The water taken for cooling is not used to produce electrical energy. During throttling in the valve, part of the energy is transferred in the form of heat to the cooling water and warms it up. The water pressure is throttled from 8.5 to 4 bars. With this comes the potential of energetic utilization.

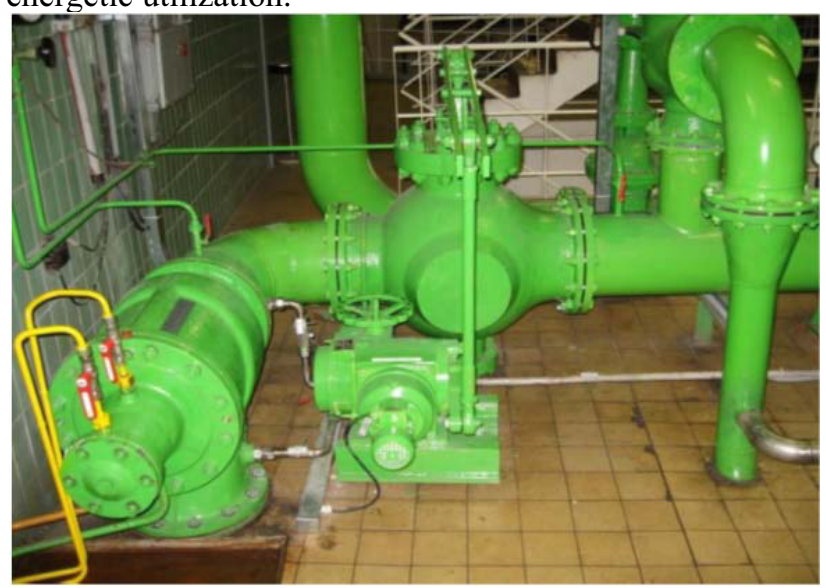

Fig. 1. Pressure reducing valve in a cooling system

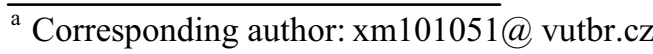

The flow is $0.145 \mathrm{~m}^{3} \mathrm{~s}^{-1}$ and the possibility of regulation of the flow to $0.08 \mathrm{~m}^{3} \mathrm{~s}^{-1}$ is required. Another utilization can be in the water mains, energetics or in the place where the conversion of energy into heat occurs due to throttling [1].

Pressure reducing valves are a source of high hydraulic losses and their replacement is possible thanks to the same resistance characteristics with the side channel turbine. Characteristics of the valve flow coefficient $\mathrm{K}_{\mathrm{v}}$ on the relative opening of the valve, see (Fig. 3.), is analogous to the turbine characteristic of unit flow $\mathrm{Q}_{11}$ on unit speed $\mathrm{n}_{11}$, see (Fig. 10.). A medium pressure drop across the valve is needed for energy recuperation.
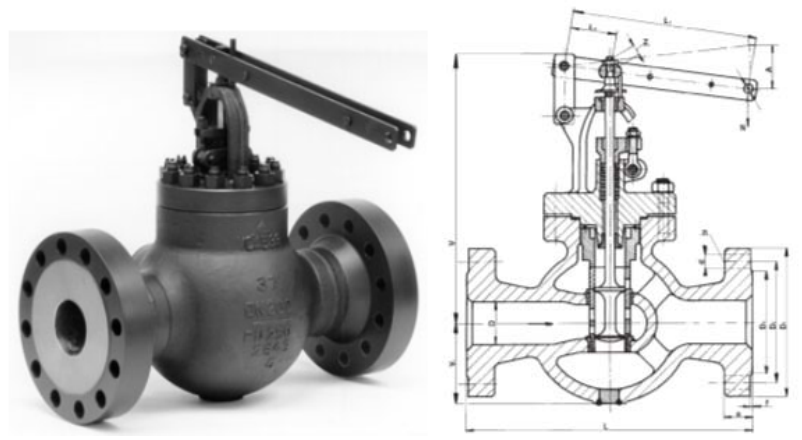

Fig. 2. Pressure reducing valve (http://www.ldmvalves.com)

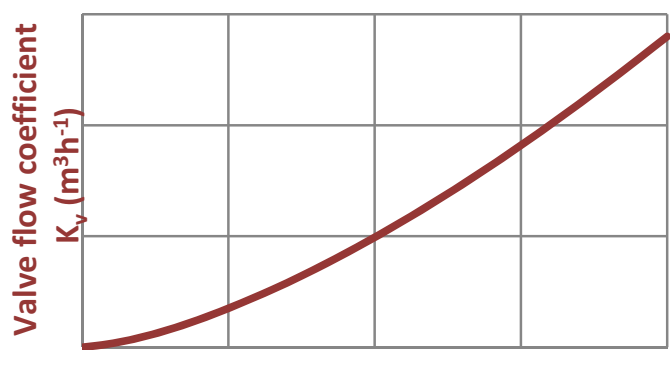

Relative opening of the valve $z(-)$

Fig. 3. Flow characteristic of a pressure reducing valve 
A further use of side channel turbines may be in the replacement of multistage pumps in turbine mode, which are not suitable for the specific speed $n_{s}<35 \mathrm{~min}^{-1}$, due to a largely problematic achievement of high efficiency. Under these parameters, a counter-rotating vortex will form in the channels between the impeller blades, disk and which will cause volume losses. This causes a significant drop in efficiency, even down to $30 \%$. In such situations, it may be advantageous to use side channel turbines as they have a higher efficiency than the lowspeed multistage pump and also a simpler construction.

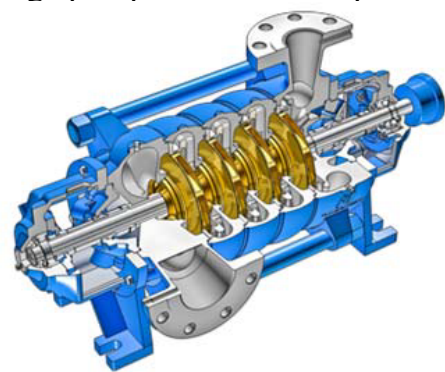

Fig. 4. Multistage pump (http://www.neptunopumps.com)

\section{Side channel machines}

Side channel machines are low-specific speed machines, which work with relatively high pressures and low flow rates and a power curve with very stable characteristics. They are smaller and simpler constructions than other comparable centrifugal pumps. The usability band with high efficiency for side channel machines is located on the border areas of hydrostatic and hydrodynamic hydraulic machines and strikes in both of these areas, see (Fig. 6.). Although side channel machines achieve relatively low efficiency, they are predominantly used for their reliability and simplicity.

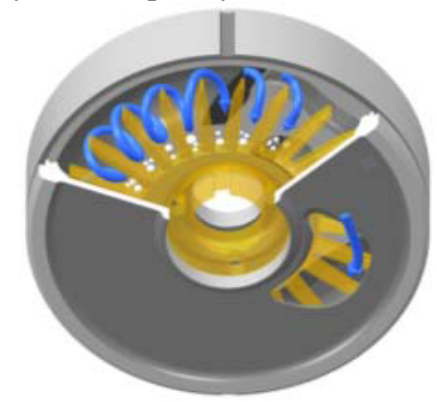

Fig. 5. Side channel machine

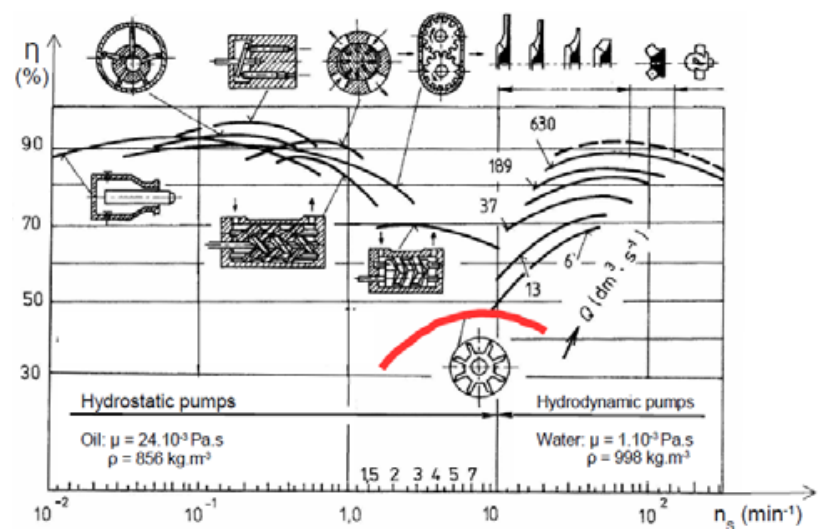

Fig. 6. Diagram of distribution of hydraulic machines according to their specific (red marked side channel machines) [2]
The design of the side channel machines is divided into two main types according to the impeller shape:

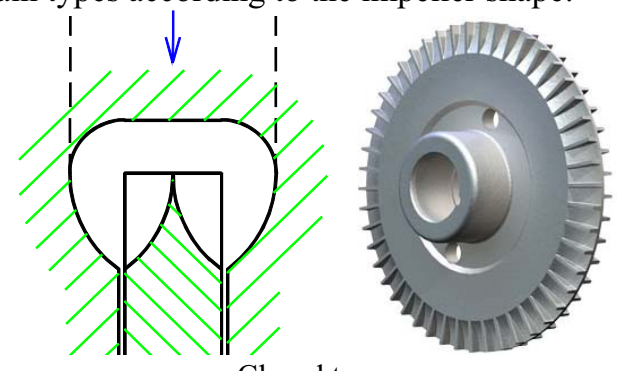

Closed type

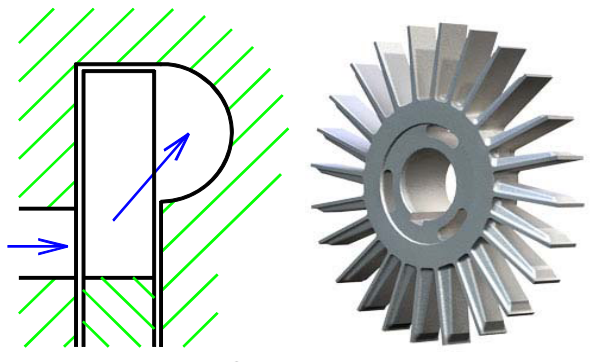

Open type

Fig. 7. The design of the impeller of side channel pumps

In the impellers of the closed type fluid is supplied directly to the channel. They are usually used for very low speed machines $\left(\mathrm{n}_{\mathrm{s}}=5 \div 10 \mathrm{~min}^{-1}\right)$, as turbines. Impellers of the open type inlet a liquid to the blades on a smaller radius, than is the radius of the channel. They are used rather in pumps because of their superior selfpriming capability.

The principle of the side channel machines is based on a huge local swirl between the blades. (Fig. 8.) shows the swirl, as obtained by experiment in PIV. (Fig. 9.) shows the swirl obtained by using the calculation software Ansys - Fluent with the model of turbulence kepsilon.

The low total efficiency achieved in side channel machines, usually from 20 to $50 \%$, is caused by several factors. The biggest influence being from hydraulic efficiency. The very principle of operation is based on the swirl and causes high power dissipation. Therefore it is not possible for the side channel machines to reach high efficiencies. Another effect of the aspirated liquid is to impact on the impeller blades and exit from the impeller to the discharge side. Volumetric efficiency affects the width of the side gap between the impeller and the stator part. The mechanical efficiency is affected by large axial forces that may occur in circulation. Suitable type of symmetrical design can minimize them. Losses in storage and mechanical seals are about the same as for similar machines.

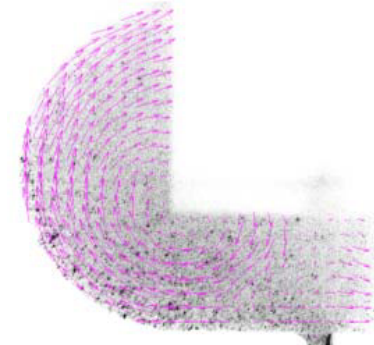

Fig. 8. Velocity field according to PIV [3]

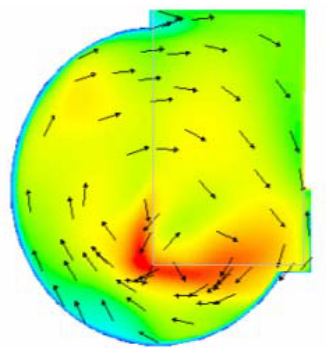

Fig. 9. Velocity field according to CFD [4] 


\subsection{Side channel turbines}

Side channel turbine falls into the category of reaction turbines. They are intended for small discharges and medium heads. The working area is within the specific speed $n_{\text {st }}=2 \div 30 \mathrm{~min}^{-1}$. The turbine can process only part of the head. The main disadvantage is low efficiency, which is usually within the range of 20 to $50 \%$. Theoretical energy efficiency characteristics $\eta$, unit flow $\mathrm{Q}_{11}$, power $\mathrm{P}_{11}$ and torque $\mathrm{M}_{11}$ are plotted in dependence on unit speed $\mathrm{n}_{11}$ in (Fig. 10.). These machines find their use due to the fact that reaching specific speed $n_{s}<35$ min $^{-1}$ with Francis' turbines at high efficiency is quite problematic. Under these circumstances, counteracting vortex starts to occur in the channels between the blades of the impeller. This causes a decrease in efficiency which thus can even get below $40 \%$. In a situation like this, it might be worth using a side channel turbine due to their higher efficiency than the low specific speed Francis' turbines. Turbines are useful for regulation due to their flat curve of efficiency in the area of optimum point and their linear dependency of flow on revolutions.

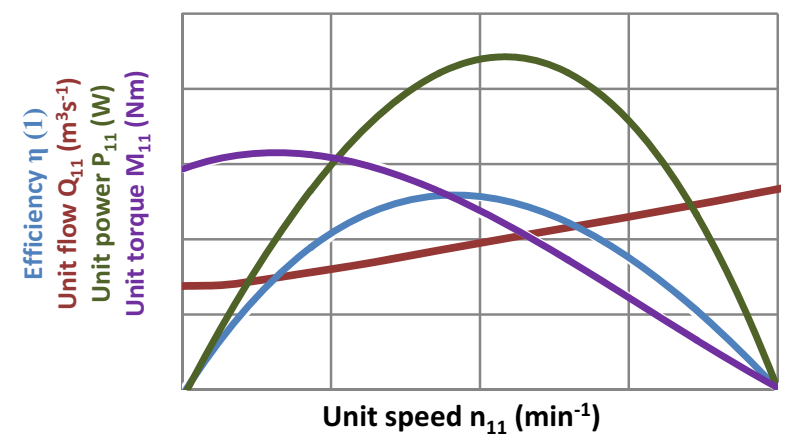

Fig. 10. The course of the energy characteristics of side channel turbines

Principle of operation of the turbine: when fluid flow occurs through the turbine runner, it is characterized by constant angular velocity. Whereas flow in the lateral channel depends on the principle of conservation of angular momentum. This causes a pressure differential between the side channels and the ends of the vanes, which causes a strong secondary liquid circulation. This movement is reinforced by circumferential movement of the blades formed by pushing on the liquid, which speeds up the entire radial width of the channel. Movement on the periphery of the runner results in a continuous shift of the difference in energy of the portion of the fluid leaving the impeller into the liquid portion in the side channels. This effect is increased due to the stroke in absence of liquid entering the side channels. Total increase in the energy of the fluid flow through the turbine is generated by pulses of exchange between the fluid in the space of the impeller and the lateral channels. The fluid passes through the runner during one revolution time [2].

Resistive characteristic of the side channel turbine the dependence of pressure drop flow $\mathrm{p}_{\mathrm{T}}$ on turbine flow $\mathrm{Q}_{\mathrm{T}}$ (Fig. 11.). Pressure drop $\mathrm{p}_{\mathrm{T}}$ is measured at the inlet and outlet of the turbine.

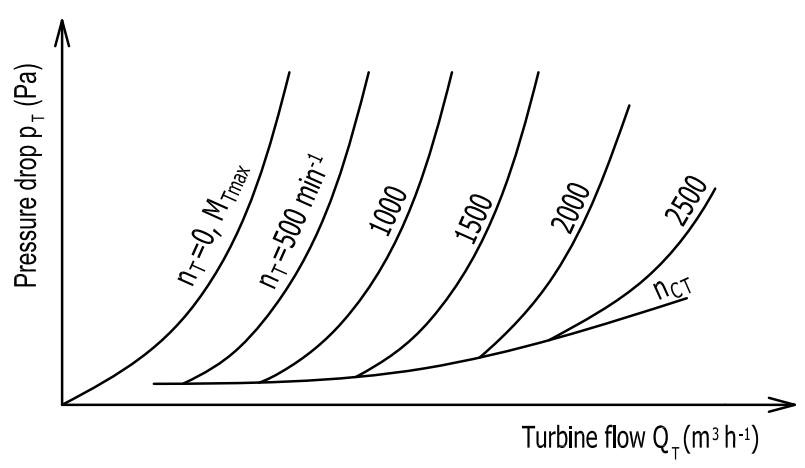

Fig. 11. Resistive characteristic of the side channel turbine [5]

\section{Side channel turbine}

\subsection{The side channel pump in turbine mode}

It is further examined, whether it is possible to use the side channel pump as a turbine for energy recovery in the piping system. For this experiment, a commercially produced side channel pump was purchased from the American company Delavan, labeled Turbo 90, see (Fig. 12.). The pump has a closed type impeller. This means that the water enters and leaks directly into the channel. The impeller is symmetrical and has 36 blades on each side. The opposing blades are rotated by half the angular pitch. The side channel is part of the iron casting cabinet, which according to the manufacturer should endure 7 bar overpressure. The size of the connecting pipe is $3 / 2$ '. The shaft is sealed with ceramic - carbon mechanical seals. The maximum speed of the wheel is $1200 \mathrm{~min}^{-1}$. The width of the side gap $\delta$ is approximately $0.1 \mathrm{~mm}$. The dimensions of the hydraulic parts are shown in (Fig. 13.). The impeller is made from nylon. It is connected with the hub straight-sided splines and can move in the axial direction. It is self-centered during operation.

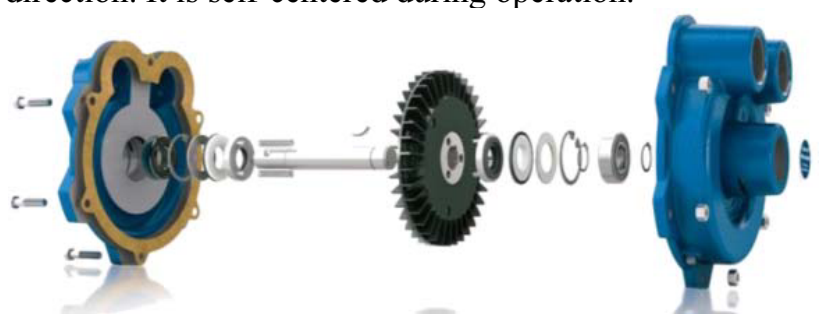

Fig. 12. Assembly of side channel machine (http://www.delavanagpumps.com)

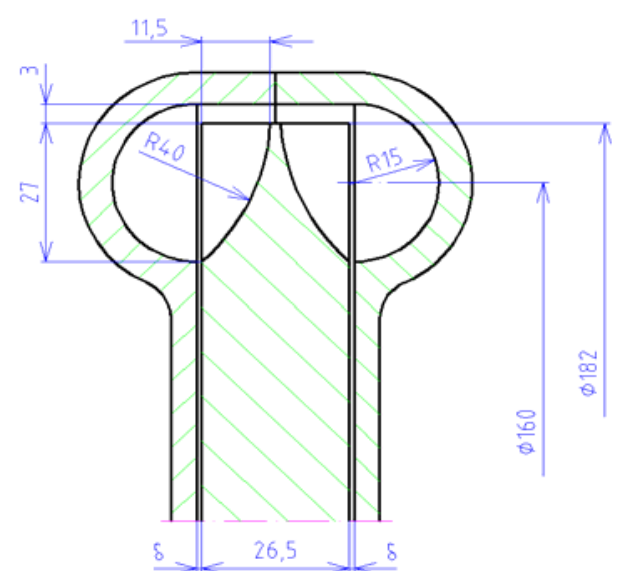

Fig. 13. Dimensions of the hydraulic shape 


\subsection{Measurement of the characteristics of the side channel turbine}

The side channel pump is measured in turbine mode. A test rig for the measurement of the energy characteristics of the side channel turbine is located in the laboratory of Victor Kaplan dept. of fluid engineering (Fig. 14. and 15.). Frequency converters are used as a generator of the change of speed and a feed pump. Values of inlet and outlet pressure, torque, speed and flow rate are recorded by an industrial computer and our program, which is written in Labview.

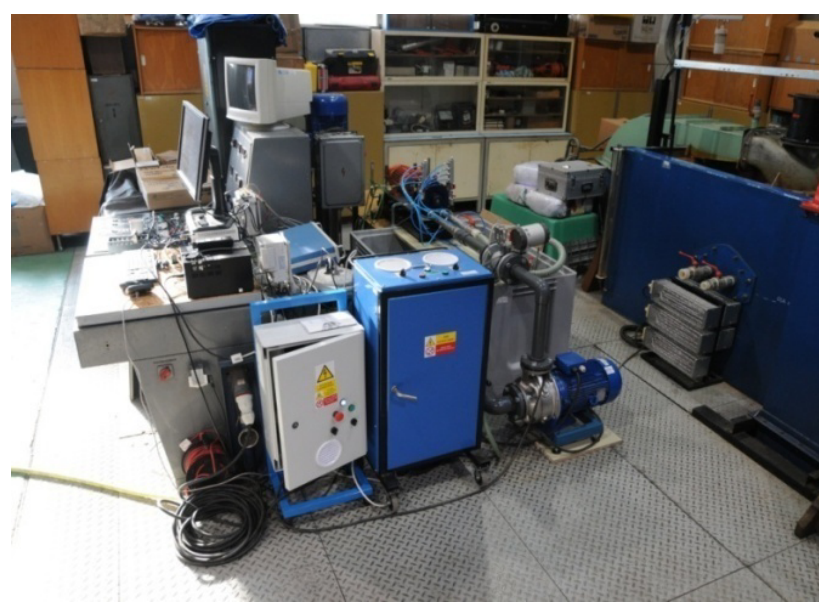

Fig. 14. Test rig

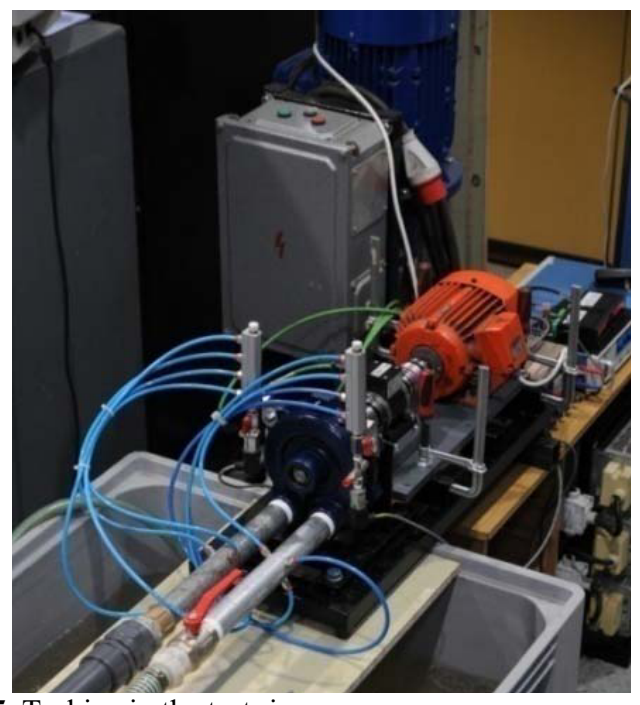

Fig. 15. Turbine in the test rig

\subsection{Characteristics of the turbine measured with a constant head}

The turbine is measured at a constant head 10, 15, 25, 35 $\mathrm{m}$ with a changing speed of the runner. At head $10 \mathrm{~m}$, the whole turbine characteristics as well as the stopped runner are re-measured. At head $35 \mathrm{~m}$, it is not possible to develop sufficient pressure by the feed pump, and therefore the characteristics are measured only partially without the optimum point and the zone of higher efficiency. The measured values are recalculated to the unit values and plotted in (Fig. 16.).
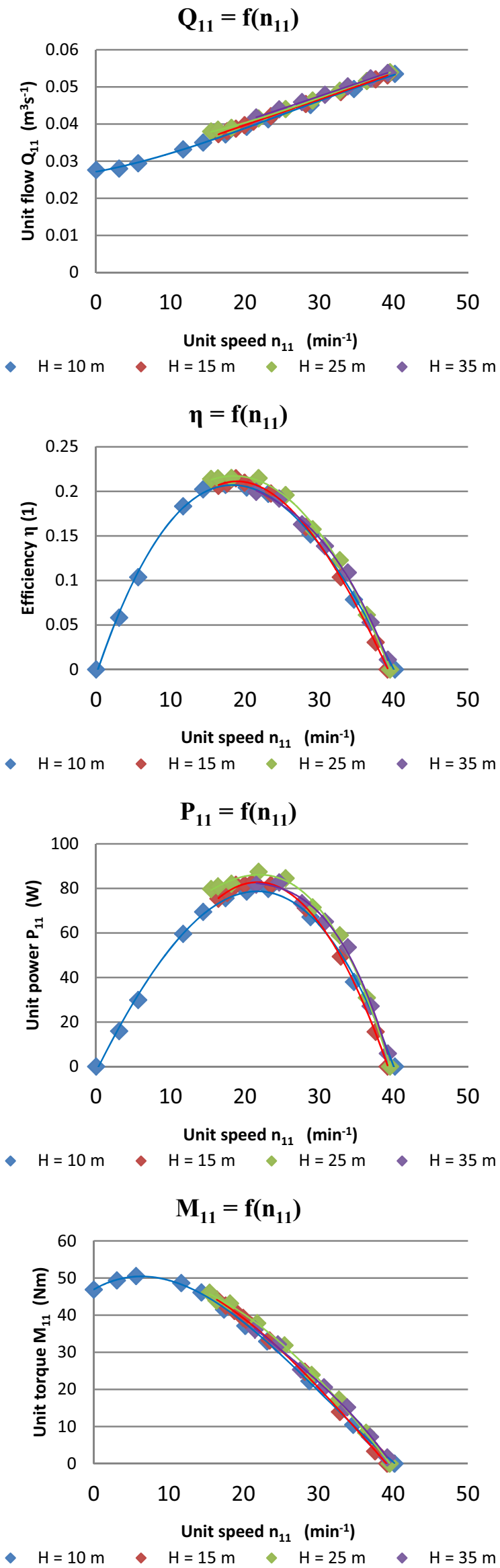

Fig. 16. Turbine graphs measured with constant heads 


\subsection{Characteristics of the turbine at constant heads with back-pressure in suction}

This section investigates the change of the characteristics of the turbine interposed in the piping system as a replacement of a pressure reducing valve. Turbine works only with a part of the pressure energy from the system. The characteristics of the turbine are measured at a constant head 15 and a changing speed of the runner. The change in the absolute values of the back-pressure in the suction line during the measurement is shown in the graph (Fig. 17.) $-\mathrm{p}_{\mathrm{b}}=\mathrm{f}\left(\mathrm{n}_{11}\right)$. The back pressure is not constant but rather depends on the flow rate. The values under backpressure are slightly lower than at atmospheric pressure on the suction.

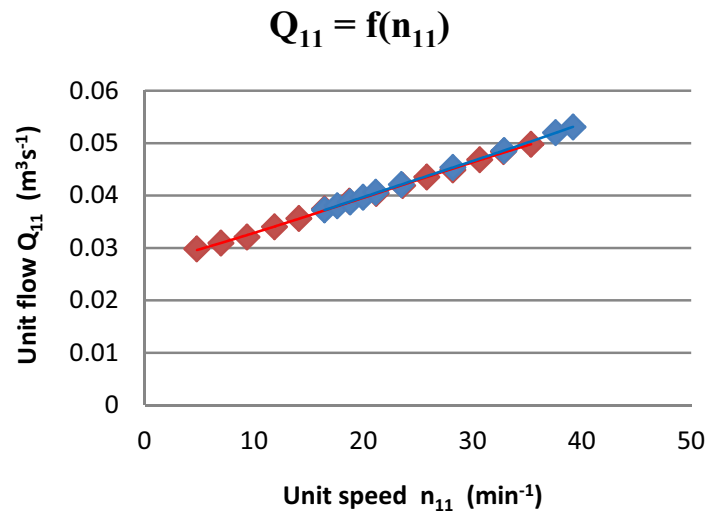

$\diamond \mathrm{H}=15 \mathrm{~m}$ with back-pressure $\quad \mathrm{H}=15 \mathrm{~m}$

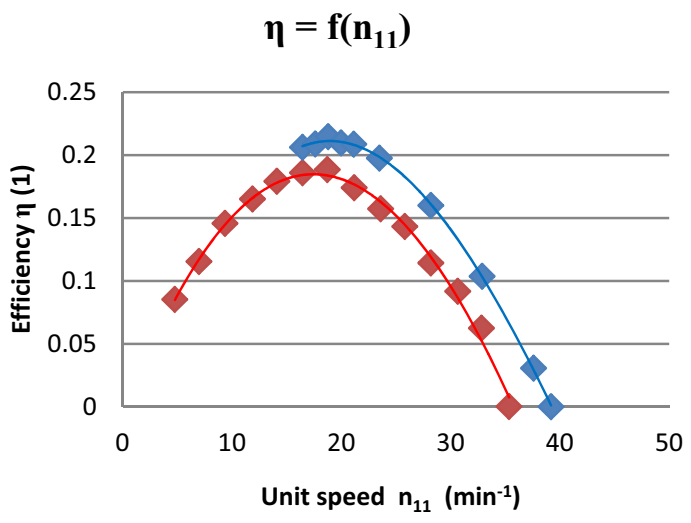

$\Delta \mathrm{H}=15 \mathrm{~m}$ with back-pressure

$\bullet \mathrm{H}=15 \mathrm{~m}$

$$
P_{11}=f\left(n_{11}\right)
$$

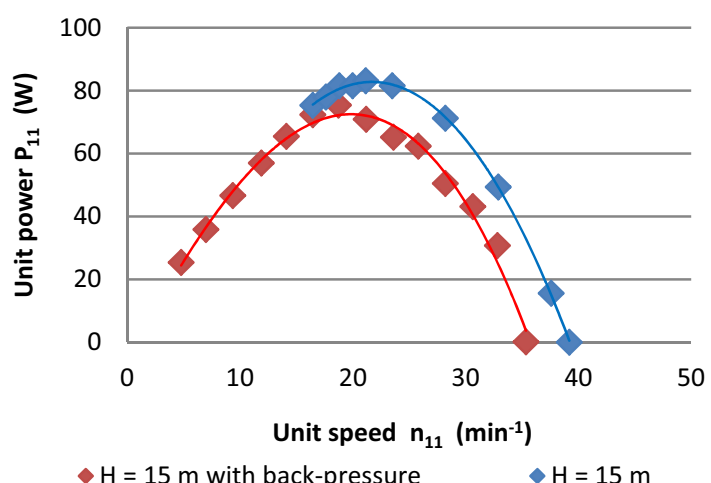

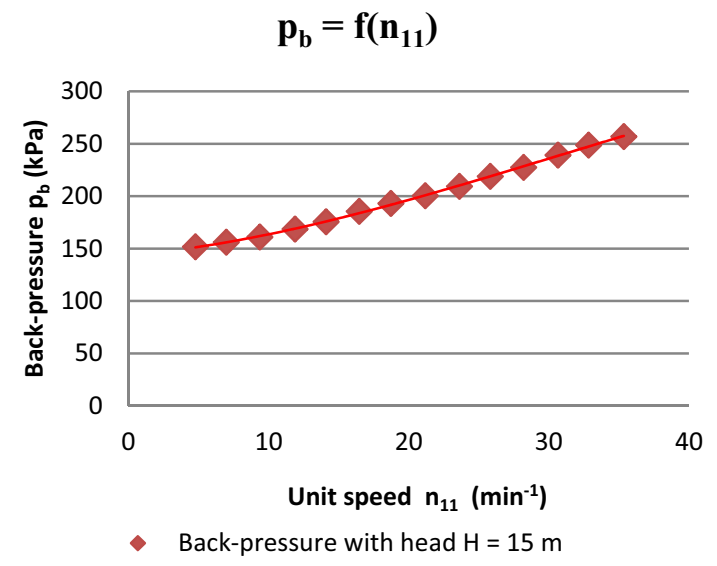

Fig. 17. Turbine graphs measured with constant heads and back-pressure in suction

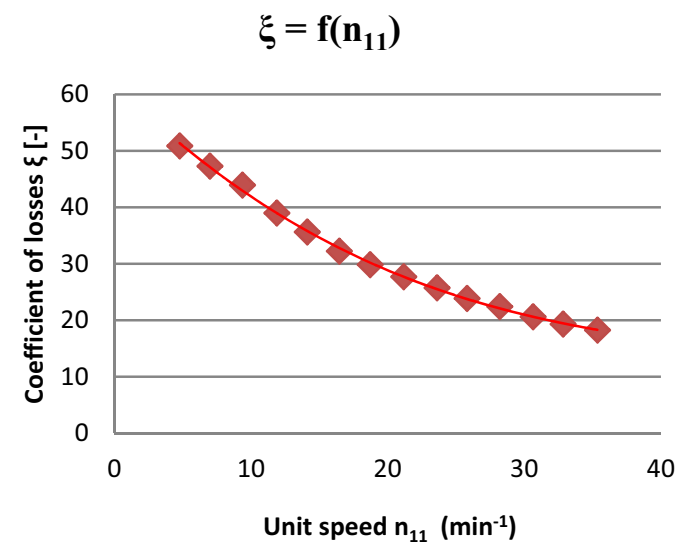

Fig. 18. Loss-making characteristic of the turbine

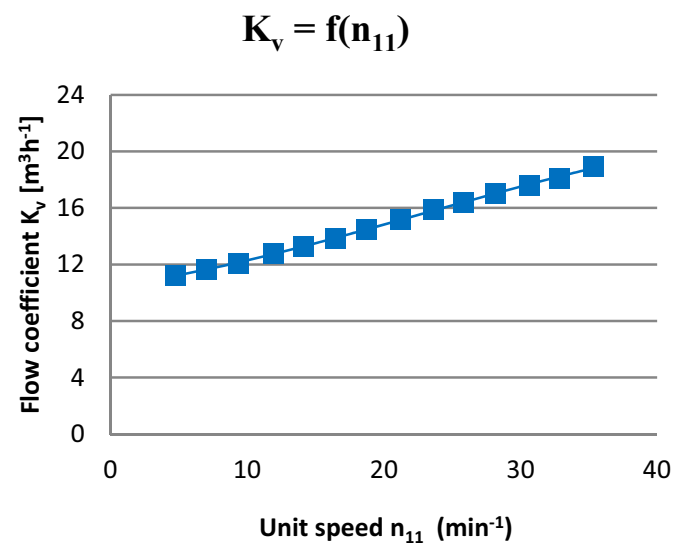

Fig. 19. Flow characteristic of the turbine

Characteristics of the turbine valve - during operation of the side channel turbine functioning as the pressure regulator, it is expected that only part of the pressure energy in the piping system will be used. Loss-making and flow characteristics of the side channel turbine Delavan Turbo 90 shown in (Fig. 12.). The characteristic curve of the turbine is the same as the curve of the pressure reducing valve. 


\section{Conclusion}

In order to verify the theory, a commercially produced side channel pump was purchased which was used to measure turbine characteristics and verify the function of the partial recovery of energy. The efficiency of recovery is about $18.9 \%$ which opens a space for optimization of the hydraulic design of the turbine valve.

\section{Notation}

$\begin{array}{ccl}\mathrm{H} & \mathrm{m} & \text { head of turbine } \\ \mathrm{K}_{\mathrm{v}} & \mathrm{m}^{3} \cdot \mathrm{h}^{-1} & \text { valve flow coefficient } \\ \mathrm{M}_{\mathrm{Tmax}} & \mathrm{N} \cdot \mathrm{m} & \text { torque of turbine } \\ \mathrm{M}_{11} & \mathrm{~N} \cdot \mathrm{m} & \text { unit torque } \\ \mathrm{n}_{\mathrm{CT}} & \min ^{-1} & \text { continuous rotation of the runner } \\ \mathrm{n}_{\mathrm{T}} & \min ^{-1} & \text { revolutions of the runner } \\ \mathrm{n}_{\mathrm{s}} & \mathrm{min}^{-1} & \text { specific speed } \\ \mathrm{n}_{\mathrm{st}} & \mathrm{min}^{-1} & \text { specific speed of turbine } \\ \mathrm{n}_{11} & \mathrm{~min}^{-1} & \text { unit speed } \\ \mathrm{p} & \mathrm{Pa} & \text { pressure } \\ \mathrm{p}_{\mathrm{b}} & \mathrm{Pa} & \text { back-pressure in suction tube } \\ \mathrm{p}_{\mathrm{T}} & \mathrm{Pa} & \text { pressure drop } \\ \mathrm{P}_{11} & \mathrm{~W} & \text { unit power } \\ \mathrm{Q} & \mathrm{m}^{3} \cdot \mathrm{s}^{-1} & \text { flow } \\ \mathrm{Q}_{\mathrm{T}} & \mathrm{m}^{3} \cdot \mathrm{s}^{-1} & \text { turbine flow } \\ \mathrm{Q}_{11} & \mathrm{~m}^{3} \cdot \mathrm{s}^{-1} & \text { unit flow } \\ \mathrm{Z} & \mathrm{m} & \text { relative opening of the valve } \\ \delta & \mathrm{mm} & \text { width of the side gap } \\ \eta & 1 ; \% & \text { efficiently } \\ \eta_{\mathrm{h}} & 1 ; \% & \text { hydraulics efficiently } \\ \mu & \mathrm{Pa} \cdot \mathrm{s} & \text { dynamic viscosity } \\ \xi & - & \text { coefficient of hydraulic losses } \\ \rho & \mathrm{kg} \cdot \mathrm{m}^{-3} & \text { density } \\ \text { Index } & & \\ \mathrm{PIV} & \mathrm{particle} & \text { image velocimetry } \\ \mathrm{CFD} & \mathrm{computational} \mathrm{fluid} \mathrm{dynamics}\end{array}$

\section{References}

1. M. Haluza, F. Pochylý, V. Habán, M. Hudec, J. Kubálek: Study of hydro-potential utilization of cooling water on the hydro-electric power plant Dalešice. Brno: Brno University of Technology, Faculty of mechanical engineering, 2007. p. 1-16.

2. P. Jandourek: Valve exploiting the principle of a side channel turbine. Thesis. Brno: Brno University of Technology, Faculty of mechanical engineering, 2014. $31 \mathrm{p}$.

3. S. Fialová: The extracorporeal blood pump. Doctoral thesis. Brno: Brno University of Technology, Faculty of mechanical engineering, 2005. $68 \mathrm{p}$.

4. Z. Dančák: Theoretical and experimental setting of the side channel pump specific energy. Brno: Brno University of Technology, Faculty of mechanical engineering, 2011, p. 90.

5. J. Bláha, K. Brada: Manual of pumping equipment. ČVUT, Prag 1997, 289 s.

\section{Acknowledgement}

This work is an output of research and scientific activities of NETME Centre, regional R\&D centre built with the financial support from the Operational Programme Research and Development for Innovations within the project NETME Centre (New Technologies for Mechanical Engineering), Reg. No. CZ.1.05/2.1.00/01.0002 and, in the follow-up sustainability stage, supported through NETME CENTRE PLUS (LO1202) by financial means from the Ministry of Education, Youth and Sports under the „National Sustainability Programme I“ and by the project of science Fund of Faculty of Mechanical Engineering of Brno University of Technology FV 16-26 „Reynold's experiment". 